Jahangiri, M.; Shahtaheri, S. J.; Adl, J. ; Rashidi, A. ; Clark, K. ; Sauvain, J. J.; Riediker, M. Emission of carbon nanofiber (CNF) from CNF-containing composite adsorbents. Journal of Occupational and Environmental Hygiene, 9(7):D130-D135, 2012.

\begin{tabular}{|l|l|}
\hline Postprint version & Final draft post-refereeing \\
\hline Journal website & $\underline{\text { http://www.tandfonline.com/loi/uoeh20 }}$ \\
\hline Pubmed link & $\underline{\text { http://www.ncbi.nlm.nih.gov/pubmed/22702232 }}$ \\
\hline DOI & $\underline{10.1080 / 15459624.2012 .691335}$ \\
\hline
\end{tabular}




\title{
Emission of carbon nanofiber (CNF) from CNF-containing composite adsorbents
}

\author{
Mehdi Jahangiri ${ }^{1}$, Seyyed Jamaleddin Shahtaheri*2 ${ }^{2}$ Javad Adl ${ }^{1}$, Alimorad Rashidi ${ }^{3}$ \\ Katherine Clark ${ }^{4}$, Jean-Jacques Sauvain ${ }^{4}$, Michael Riediker ${ }^{4}$ \\ ${ }^{1}$ Department of Occupational Health, School of Public Health, Tehran University of Medical Sciences, \\ Tehran, Iran \\ ${ }^{2}$ Department of Occupational Health, School of Public Health, Center for Environmental Research, \\ Tehran University of Medical Sciences, Tehran, Iran \\ ${ }^{3}$ Research Institute of Petroleum Industry (RIPI), Tehran, Iran \\ ${ }^{4}$ Institute for Work and Health, Lausanne, Switzerland
}

Word count: 2216

* Corresponding author: shahtaheri@tums.ac.ir Tel: +989121779019, Fax: +982188951390 


\begin{abstract}
In spite of numerous applications of carbon nanofibers (CNFs) in a variety of fields, the potential release of airborne CNF during their special application, which could lead to workers or end-users exposure, has not been well investigated. In this study, the potential release of CNF from an organic vapour respirator cartridge was evaluated by carbon analysis and microscopy analysis. The cartridge consisted of an AC (Activated Carbon)/CNF composite adsorbent and different types of particulate filters. The composite adsorbent CNF were prepared by chemical vapour deposition (CVD).

Air was passed through the prepared cartridge for 12 hours at $12 \mathrm{l} / \mathrm{min}$ and particles were collected on sampling filters suitable for measuring organic and elemental carbon (OC/EC) by carbon analysis based on the NIOSH 5040 method. Breakthrough of CNFs was also checked by scanning and transmission electron microscopy (SEM/TEM).

This study found only minimal amounts of released elemental carbon while passing the air through the cartridge. Meanwhile TEM photos showed a few CNF structures for AC/CNF composite adsorbents which were not in the critical range in terms of length, aspect ratio, or number.
\end{abstract}

Keywords: Carbon nanofiber release, Respirator cartridge, Composite adsorbent 


\section{INTRODUCTION}

Carbon nanofibers (CNFs) are a new class of synthesized carbonaceous materials which have received growing attention and been extensively studied due to their interesting properties (i.e., purity, mechanical strength, high geometric surface area) and potential for use as adsorbents and catalyst supports. ${ }^{(1-4)}$. Production costs of CNF are significantly lower than those of carbon nanotubes (CNT) and they still provide high performance for certain applications ${ }^{(5,6)}$.

CNFs are produced from the catalytic decomposition of hydrocarbon gases or carbon monoxide over selected metal particles - including iron, cobalt, nickel and some of their alloys - at temperatures in the range of $400-1000^{\circ} \mathrm{C}$. This process is called "Chemical Vapor Deposition (CVD)" (5).

CNFs are characterized by the graphite-like structure at the nano- scale. Variable alignments of laminated hexagonal layers along the fiber axis typically provide three types of CNFs: platelet (aligned perpendicular to the fiber axis), tubular (aligned parallel to the fiber axis), and herringbone (aligned at an angle to the fiber axis) ${ }^{(2)}$. These structures differ according to the growing conditions and the metal used as a catalyst ${ }^{(7)}$.

Several studies have been conducted in the use of CNF with improved adsorbent properties. For example, Lim et al ${ }^{(3)}$ gave Activated carbon fiber (ACF) multiple additional properties by growing CNFs on its catalytic surface. This improved the performance of ACFs for such applications as SOx and NOx removal. Schlogl et $\mathrm{al}^{(8)}$, grew carbon nanostructures (CNFs and CNTs) on a carbonaceous carrier (activated carbon-AC) for the removal metal species in water purification. In a recent study by the authors $^{(9)}$, a composite adsorbent AC and CNF was prepared in granular form and employed successfully in organic respirator cartridges, for the removal of organic vapours. In the prepared $\mathrm{AC} / \mathrm{CNF}$ composite adsorbent, CNF were selectively deposited in the micropores of the AC catalyst particles by CVD.This study $^{(9)}$ suggested that the granular form of AC/CNF composite could be a efficient alternative adsorbent for respirator cartridges due to its larger adsorption capacities and lower weight.

The broad utility of manufactured nanomaterials is resulting in increased levels of production, greater risk of human exposure and more potential for release of these novel materials into the environment. Therefore, close attention to risks - i.e. the potential for exposure and toxic responses to manufactured nanoparticles, including various fibrous nanomaterials, is very important ${ }^{(10)}$. A growing body of literature indicates a potential hazard from exposure to carbon nanofibers ${ }^{(5,11-13)}$. For example, one study showed that CNFs could penetrate human cells in target organs and cause cellular damage ${ }^{(12)}$ - Kisin et al, showed that CNFs could have a genotoxicity effect comparable to asbestos and stronger than Singlewalled carbon nanotubes (SWCNT) ${ }^{(6)}$. Due to the huge number of potential applications for $\mathrm{CNF}^{(14)}$, 
their possible release during fabrication or use needs to be better investigated. In this study, the potential release of $\mathrm{CNF}$ from a respirator cartridge prepared from $\mathrm{AC} / \mathrm{CNF}$ composite adsorbent and different particulate filters was evaluated by carbon analysis and electron microscopy analysis.

\section{MATERIAL AND METHODS}

\section{Preparation of Cartridge}

The experimental cartridge was designed to correspond to an AC/CNF composite adsorbent prepared for the purpose of respiratory protection against organic vapours. For this purpose, $12 \mathrm{~g}$ of granular CNF/AC absorbent ( $40 \% \mathrm{CNF}$ ) was placed in a cartridge holder (a plastic case) and retained in it with different particulate filters. Four different types of particulate filters commonly used in respirators were used in these studies: coarse, pre-filter (3M 6075), FFP2 (3M 9322) and FFP3 (3M 9332). The amount of adsorbent in the cartridge holder and the air flow rate which passed through it was proportional to that of a typical respirator cartridge $\left(25 \mathrm{~g}-40 \mathrm{~g}\right.$ and $\left.301 / \mathrm{min}^{(15,16)}\right)$. This cartridge was connected to a sampler equipped with a quartz particle filter used to determine the elemental carbon (EC) and organic carbon (OC) present in the potentially released particles (see Figure 1). All the system was connected to a high volume flow pump, running at 12 1/min for 12 hours.

\section{Carbon Analysis}

Elemental carbon has been proposed as an indicator for exposure assessment with carbon nanomaterials ${ }^{(17-19)}$. In this study, the release of carbonaceous particles (CNFs and/or AC) from the composite adsorbent was measured as elemental carbon (EC) by carbon analysis. Air passed through the cartridge holder (including adsorbents and different particulate filters) and afterwards through $37 \mathrm{~mm}$ quartz filters (in a 3-piece, $37 \mathrm{~mm}$ sampler cassette) using the above experimental set up. For each type of particulate filter, three experiments were done.

To assess the potential contribution of EC present in the laboratory's ambient air, air was also passed through the particulate filters (pre and FFP2 filters) without adsorbents in the cartridge. These experiments were done in parallel with the samples collected after passing the cartridge (adsorbents and different particulate filters).

Collected particles on quartz filters were analyzed by a thermal/optical aerosol carbon monitor (Sunset Laboratory, OCEC analyzer, OR, USA) according to NIOSH analytical method 5040 for airborne EC ${ }^{(19}$, 20)).

In this carbon analysis, a standard sized punch $\left(1.5 \mathrm{~cm}^{2}\right)$ was made from a previously collected quartz filter sample. This was placed in a quartz oven and first heated under an oxygen-free atmosphere (He 99.999\%) to remove organic carbon (OC), then heated again and the EC is oxidized. In the NIOSH method, the $\mathrm{CO}_{2}$ resulting from OC or EC decomposition is catalytically converted to $\mathrm{CH}_{4}$. The methane is subsequently measured using a flame ionization detector (FID). Additionally, the transmittance of a 
laser beam through the filter sample is recorded in order to take into account possible pyrolysis of the organic material. The point at which the value of transmittance becomes the same as the initial value is defined as "the split" between $\mathrm{OC}$ and $\mathrm{EC}$. The $\mathrm{CO}_{2}$ produced before the split is defined as $\mathrm{OC}$, and after the split is defined as $\mathrm{EC}^{(21,22)}$.

The minimum detection limit of this analyzer was $0.15 \mu \mathrm{g} / \mathrm{cm}^{2}$, corresponding to $0.15 \mu \mathrm{g} / \mathrm{m}^{3}$ in our experimental conditions. The results obtained from the instrument, were corrected for potential OC and EC contamination on the filters by subtraction of results from an unused filter. Then, the mean concentration of OC and EC was calculated by dividing the mass of carbon (OC or EC) by the total volume of air which passed through the filter during the period of sampling.

\section{Microscopy Analysis}

Qualitative assessment for determining the breakthrough of CNF through the adsorbents was done by scanning electron microscopy (SEM) and transmission electron microscopy (TEM). Using the above experimental set up in different experiments, filter-based air samples were collected on $25 \mathrm{~mm}$ MFMillipore Membrane, mixed cellulose esters (MCE) and $25 \mathrm{~mm}$ gold-coated polycarbonate membrane filter (in carbon-filled conductive polypropylene sampler cassettes) for TEM (Philips CM-100 operated at $100 \mathrm{kV})$ and SEM (JSM Jeol-6300F operated at 10kV) respectively.

The MEC filters were transferred on the TEM grids, dissolved by dimethylformamide and acetic acid and treated in the plasma furnace according to ISO standard $10312^{(23)}$ for filter based asbestos exposure assessment. The observations were done with a 20,000:1 magnification. Two samples per filter (one on a copper grid and one on a gold grid) were observed and 100 randomly selected grid fields in each sample were checked for CNFs. All experiments for assessing breakthrough of CNFs were conducted in triplicate for each type of particulate filter using TEM/SEM.

The calculation of average CNF fiber/agglomerate concentration in TEM grids was done by fiber counting according to the following formula:

$\mathrm{C}=\mathrm{Af} \times \mathrm{E} /(\mathrm{Ag} \times \mathrm{k} \times \mathrm{V} \times 1000)^{(23)}$

C - Concentration of fibres per $\mathrm{cm}^{3}$

Af - Active surface of the filter $\left[\mathrm{mm}^{2}\right]\left(420 \mathrm{~mm}^{2}\right)$

Ag - Surface of one TEM squares $\left[\mathrm{mm}^{2}\right]\left(0.011 \mathrm{~mm}^{2}\right)$

E - Number of fibres counted

$\mathrm{k}$ - Number of TEM squares counted

$\mathrm{V}$ - Volume of the sampled air

The extrapolated number of fibres was based on the size of one grid: $0.011 \mathrm{~mm}^{2}$ and total filter surface of $420 \mathrm{~mm}^{2}$ for 14 hours sampling with a flow rate of $12 \mathrm{l} / \mathrm{m}$.

The gold filters were transferred to the grid directly and observed by SEM 


\section{Carbon Analysis}

Figure 2 (left) shows the amount of OC and EC measured after passing air through the AC/CNF adsorbents and different particulate filters.

As can be seen, all of particulate filters (coarse, pre-filter, FFP2 and FFP3) did not show any detectable amounts of released EC (below the detection limit of $0.15 \mu \mathrm{g} / \mathrm{m}^{3}$ ).

Figure 2 (right) shows the amount of measured OC and EC after passing air through pre and FFP2 particulate filters, with and without CNF-containing adsorbents. As can be seen, interestingly the amount of OC and EC for the samples after passing air through pre and FFP2 filters (without adsorbents) are higher than the samples collected after passing air through the adsorbents. This is probably because some of the $\mathrm{OC}$ and $\mathrm{EC}$ in the ambient air have been removed while passing through the adsorbents.

Figure 2 also shows that there are some organic carbons in the filters analyzed. This may suggest that some volatile organic material got absorbed on the surfaces of adsorbents and filter fibers, either during production or storage of these materials, which then led to these small amounts of OC on the filters. However, when considering the values measured from collected samples after passing the air through the particulate filters (without adsorbents- Figure 2), a large part of the measured OC values could be attributed to the laboratory's ambient air.

\section{TEM and SEM Observations}

Figure 3 shows the TEM photographs of the original synthesized AC/CNF composite adsorbents which were used in this study. As can be seen, CNFs with a very thin diameter of about 10-20 nm were formed uniformly on the AC. Many CNFs are intertwined. Such thin CNFs are not of a tubular type but are closer to a herringbone type.

Figure 4 shows the TEM photographs of samples collected on MCE filters after passing air through the cartridge including CNF-containing composite adsorbents and (a) coarse, (b) pre, (c) FFP2 and (d) FFP3 particular filters. We can observe some fibrous agglomerated structures in the photographs for the coarse and pre-particulate filters. It appears that the prepared composite adsorbents released small amounts of CNF which these two types of particulate filters could not retain completely.

As Figure 4 shows, the size of these fibers is significantly lower than that used to define asbestos fibers (length $\geq 5 \mu \mathrm{m}$ and aspect ratio $\geq 3: 1^{(23)}$ ), so CNF cannot be considered an analogous hazard to asbestos fibers in terms of penetration into the lung wall ${ }^{(24)}$. Furthermore, the average concentration of CNF fiber/agglomerate in TEM grids after passing air through CNF-containing adsorbents was $1.32 \mathrm{E}^{-4}$ and $4.41 \mathrm{E}^{-5}$ fibers $/ \mathrm{ml}$, for coarse and pre particulate filters respectively. This is significantly lower than the recommended occupational exposure limits in Switzerland for carbon nanotubes and carbon nanofibers (0.01 fibers $/ \mathrm{ml}$; this limit is the same as for asbestos fibers ${ }^{(24)}$ ). 
Figure 5 shows scanning electron micrographs of collected samples on the gold-coated filters after passing air through the cartridge including CNF-containing composite adsorbents and (a) coarse, (b) pre, (c) FFP2 and (d) FFP3 particular filters. No fiber structures were observed on these SEM monograms. The number of agglomerated fibers counted on TEM samples collected after the coarse particulate filter was higher than after the pre-filter. Despite this (low) number of CNF structures being detectable on the TEM grid, SEM photos didn't show any CNFs structures even for coarse particulate filter (Figure 5). For the FFP2 and FFP3 filters, we did not detect any fiber structured object on TEM grids. These results were in agreement with carbon analysis EC measurements.

\section{CONCLUSION}

This study found no release of CNF from fully intact filter cartridges containing AC/CNF composite adsorbent, and only minimal amounts if the cartridges were missing the high efficiency filters they usually contain. The carbon analysis experiments in this study showed a small amount of EC after passing air through the cartridge including CNF-containing composite adsorbents and coarse and preparticulate filter, which could be attributed to the EC in ambient air. Moreover this amount of EC is significantly lower than NIOSH's recommended occupational exposure limits for carbon nanotubes and nanofibers ${ }^{(19)}$. Meanwhile TEM photographs showed a few CNF structures for AC/CNF composite adsorbents with coarse and pre particulate filters which were not in the range defined for asbestos fibers in terms of length and aspect ratio. Therefore, it is concluded that the amount of CNF released from CNFcontaining composite adsorbents prepared by a CVD process followed by a heating process (as a fixation step) is negligible. This is probably because CNFs are solidly bound to the AC by the process they undergo during synthesis, in which contaminating catalytic metals are frequently removed either by high temperature vaporization or acid treatment ${ }^{(19)}$. It seems that the synthesis process of heating the composite adsorbents at $550^{\circ} \mathrm{C}$ under flowing nitrogen gas, has acted as a fixation step, stabilizing the catalyst precursor particles. Consequently this has controlled and stabilized nanofiber growth on the activated carbon. ${ }^{(8)}$. In this study the release of CNFs from AC/CNF composite adsorbents was evaluated under conditions equivalent to the normal use of a respirator cartridge in the workplace. Further studies are needed to evaluate the potential release of CNFs in different degradation situations e.g. chemical exposition, climatic conditions and shipping conditions. These efforts should be made to ensure that the cartridges are safe by design, before the production stage and also to prevent the emission of CNFs during respirator use. 


\section{ACKNOWLEDGEMENTS}

This research was supported by Tehran University of Medical Sciences grant No. 10142-27-01-89. The work was carried out at the Institute for Work and Health (IST), Universities of Lausanne and Geneva, Switzerland. Hence, the authors would like to acknowledge both Teheran University and IST for their valuable support.

\section{REFRENCES}

1.Iijima, S.: Helical microtubules of graphitic carbon. Nature 354(56-58.)(1991).

2.Rodriguez, N.M., A. Chambers, and R.T.K. Baker: Catalytic Engineering of Carbon Nanostructures. Langmuir 11(10): 3862-3866 (1995).

3.Seongyop Lim, S.-H.Y., Yoshiki Shimizu, Hangi Jung, and Isao Mochida: Surface Control of Activated Carbon Fiber by Growth of Carbon Nanofiber. Langmuir 20: 5559-5563 (2004).

4.Jahangiri, M., S.J. Shahtaheri, J. Adl, A. Rashidi, H. Kakooei, and A.R. Forushani: The adsorption of benzene, toluene and xylenes (BTX) on the carbon nanostructurs: the study of different parameters. Fresenius Environmental Bulletin 20 (4a): 1036-1045 (2011).

5.Genaidya, A., T. Tolaymatb, R. Sequeiraa, M. Rinderc, and D. Dionysioua: Health effects of exposure to carbon nanofibers: Systematic review, critical appraisal, meta analysis and research to practice perspectives. Science of the Total Environment 407: 3686 - 3701 (2009).

6.Kisin, E.R., A.R. Murray, L. Sargent, D. Lowry, M. Chirila, K.J. Siegrist et al.: Genotoxicity of carbon nanofibers: Are they potentially more or less dangerous than carbon nanotubes or asbestos?

Toxicology and Applied Pharmacology 252(1): 1-10 (2011).

7.Serp, P., M. Corrias, and P. Kalck: Carbon nanotubes and nanofibers in catalysis. Appl. Catal. A 253: 337 (2003).

8.Schlogl, R.: Nanocarbon-activated carbon composite. In US patent. United States.: Sud-Chemie AG, Munich (DE), 2009.

9.Jahan-Giri, M., S.J. Shahtaheri, J. Adl, A. Rashidi, A. Ghorbanali, H. Kakooei et al.: Preparation of new adsorbents from Activated Carbon and Carbon Nanofiber (AC/CNF) for manufacturing organicvapour respirator cartridge. Accepted for Iranian Journal of Environmental Health Science \& Engineering, In press.

10.Shvedova, A.A., V.E. Kagan, and B. Fadeel: Close encounters of the small kind: adverse effects of man-made materials interfacing with the nano-cosmos of biological systems. Annu. Rev. Pharmacol.

Toxicol. 50: 63-88 ( 2010).

11.Magrez, A., S. Kasas, V. Salicio, N. Pasquier, J.W. Seo, M. Celio et al.: Cellular Toxicity of Carbon-Based Nanomaterials. Nano Letters 6(6): 1121-1125 (2006).

12.Genaidya, A., R. Sequeira, M. Rinder, and A. A-Rehim: Risk analysis and protection measures in a carbon nanofiber manufacturing enterprise: An exploratory investigation. Science of the Total

Environment 407: 5825-5838 (2009).

13.Yokoyama, A., Y. Sato, Y. Nodasaka, S. Yamamoto, T. Kawasaki, M. Shindoh et al.: Biological Behavior of Hat-Stacked Carbon Nanofibers in the Subcutaneous Tissue in Rats. Nano Letters 5(1): 157 161 (2004).

14.Enanonewsletter: Carbon nanotubes, 2008.

15.Nelson, G.O., and A.N. Correia: Respirator cartridge efficiency studies: VIII. summary and conclusions. American Industrial Hygiene Association Journal 37(9): 514 - 525 (1976).

16.EN141;2000: "Respiratory Protective Device-Gas filters and combined filters- Requirement, testing, marking." 2000.

17.Kuhlbusch TAJ, and F. H: Particle characteristics in the reactor and palletizing areas of carbon black production. Occup Environ Hyg 3: 558-567 (2006). 
18.Kuhlbusch TAJ, Neumann S, and F.H. . Number size distribution, mass concentration, and particle composition of PM1, PM2.5, and PM10 in bag filling areas of carbon black production. J Occup Environ Hyg 1:660-671. 1(2004).

19.NIOSH: Occupational Exposure to Carbon Nanotubes and Nanofibers, NIOSH Current Intelligence Bulletin, 2010.

20.NIOSH: "Elemental Carbon (Diesel Exhaust) NIOSH Manual of Analytical Methods (NMAM), Method 5040. ." Cincinnati, Ohio: U.S. Department of Health and Human Services,Centers for Disease Control and Prevention, 1999.

21.Birch, M.E., and R.A. Cary: Elemental Carbon-based method for monitoring occupational exposures to particulate diesel exhaust. Aerosol Sci Technol 25: 221-224 (1996).

22.Mariko, O.-O., and J.S. Thomas: Diesel Exhaust Particles in the Work Environment and their Analysis. Industrial Health 42: 389-399 (2004).

23."BS ISO 10312:1995 Ambient air. Determination of asbestos fibres. Direct-transfer transmission electron microscopy method." 1995.

24.Suva: Valeurs limites d'exposition aux postes de travail 2011: Suva, Médecine du travail, 2011. 


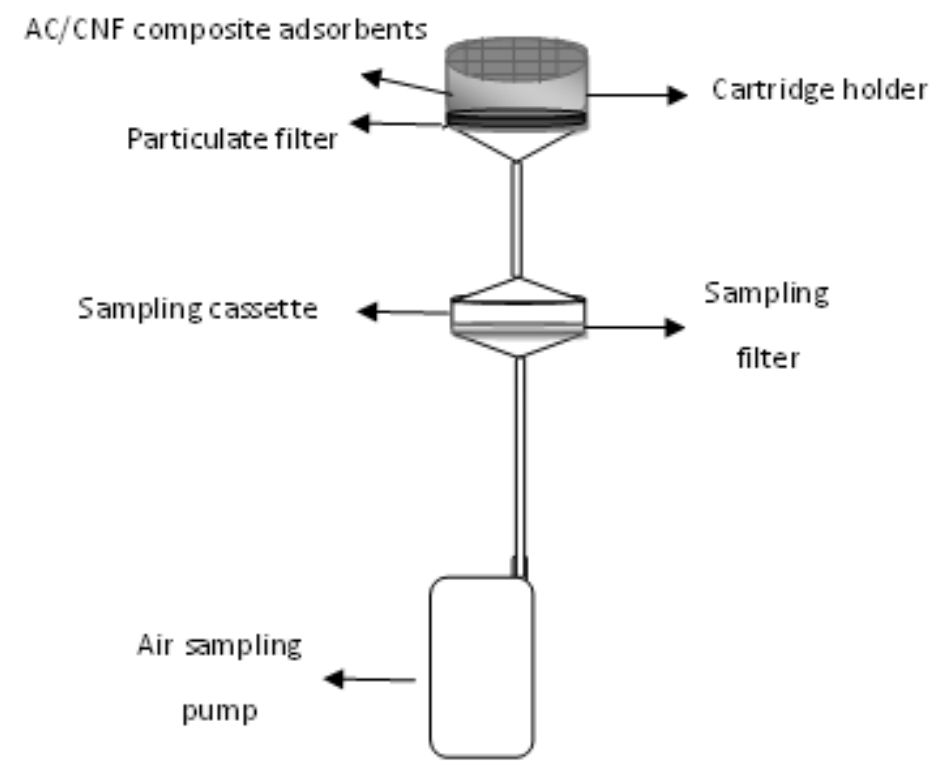

FIGURE 1 Schematic diagram of the experimental set up for evaluation of CNF breakthrough experiments 

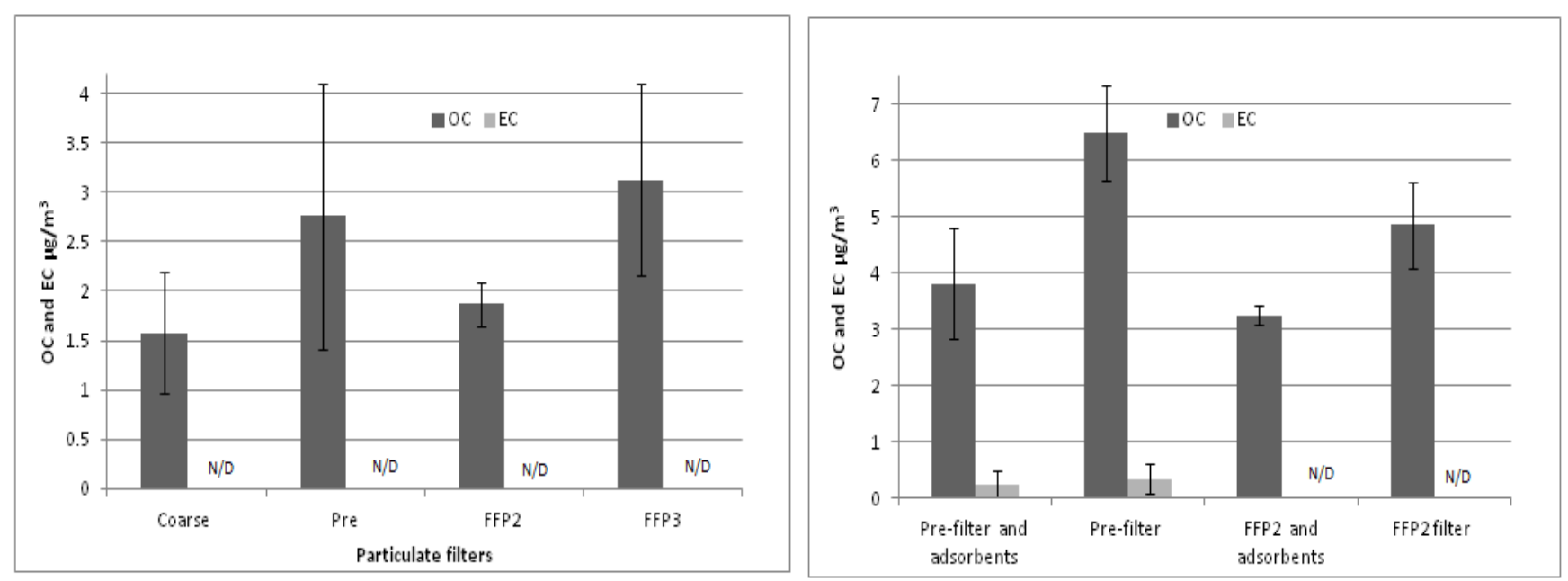

FIGURE 2 Amount of OCEC after passing air through AC/CNF adsorbents and different particulate filters (left) Amount of OCEC after passing air through pre and FFP2 filters with and without adsorbents. Error bars have been calculated based on standard deviations. N/D; Not detected (below the detection limits)

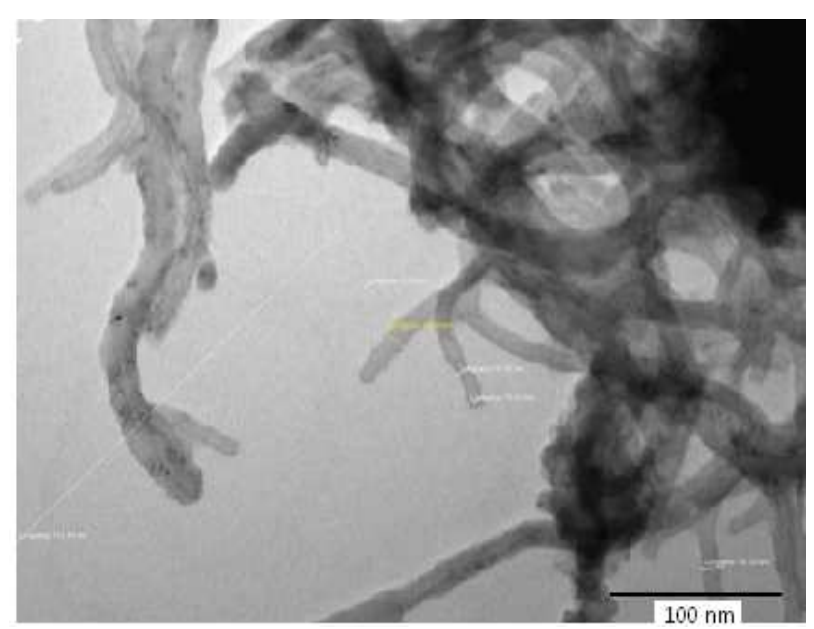

FIGURE 3 TEM photograph of synthesized AC/CNF composite 

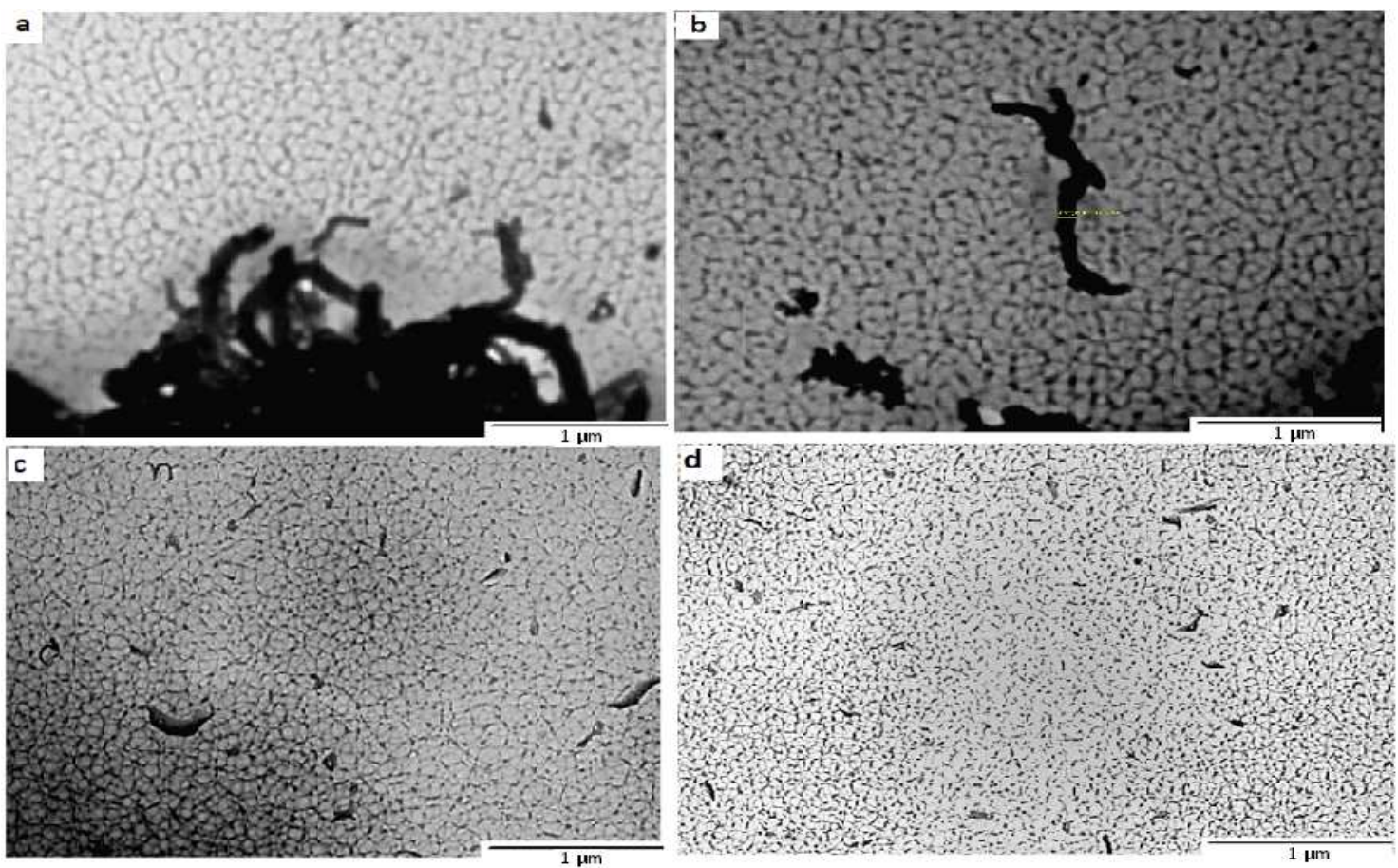

FIGURE 4 TEM photographs of collected samples on MCE filters after passing air through cartridges including CNFcontaining composite adsorbents and (a) coarse, (b) pre, (c) FFP2 and (d) FFP3 particular filters. CNF structures are observed in photographs (a) and (b).
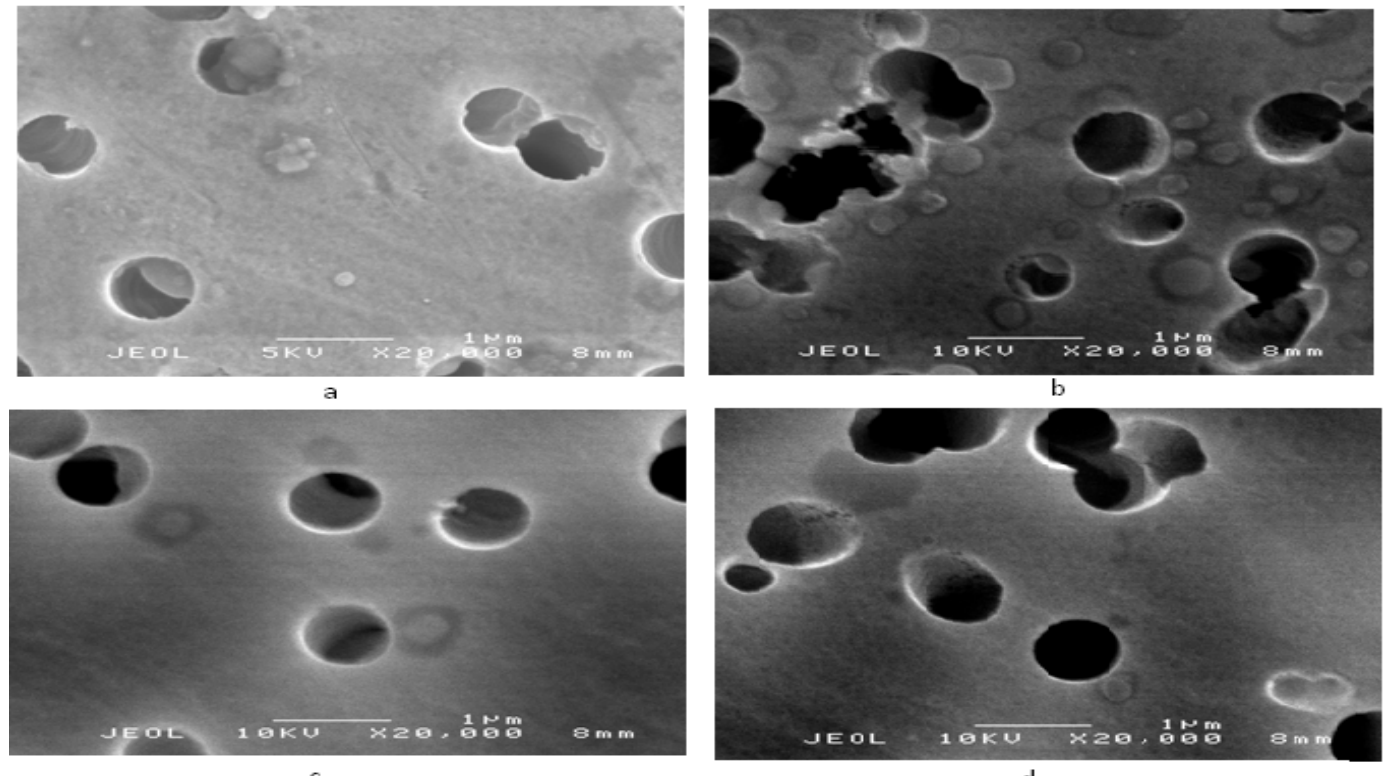

FIGURE 5 SEM photographs of samples collected on MCE filters after passing air through the cartridge including CNFcontaining composite adsorbents and (a) coarse, (b) pre, (c) FFP2 and (d) FFP3 particular filters. No CNF structures are observed on these pictures. 